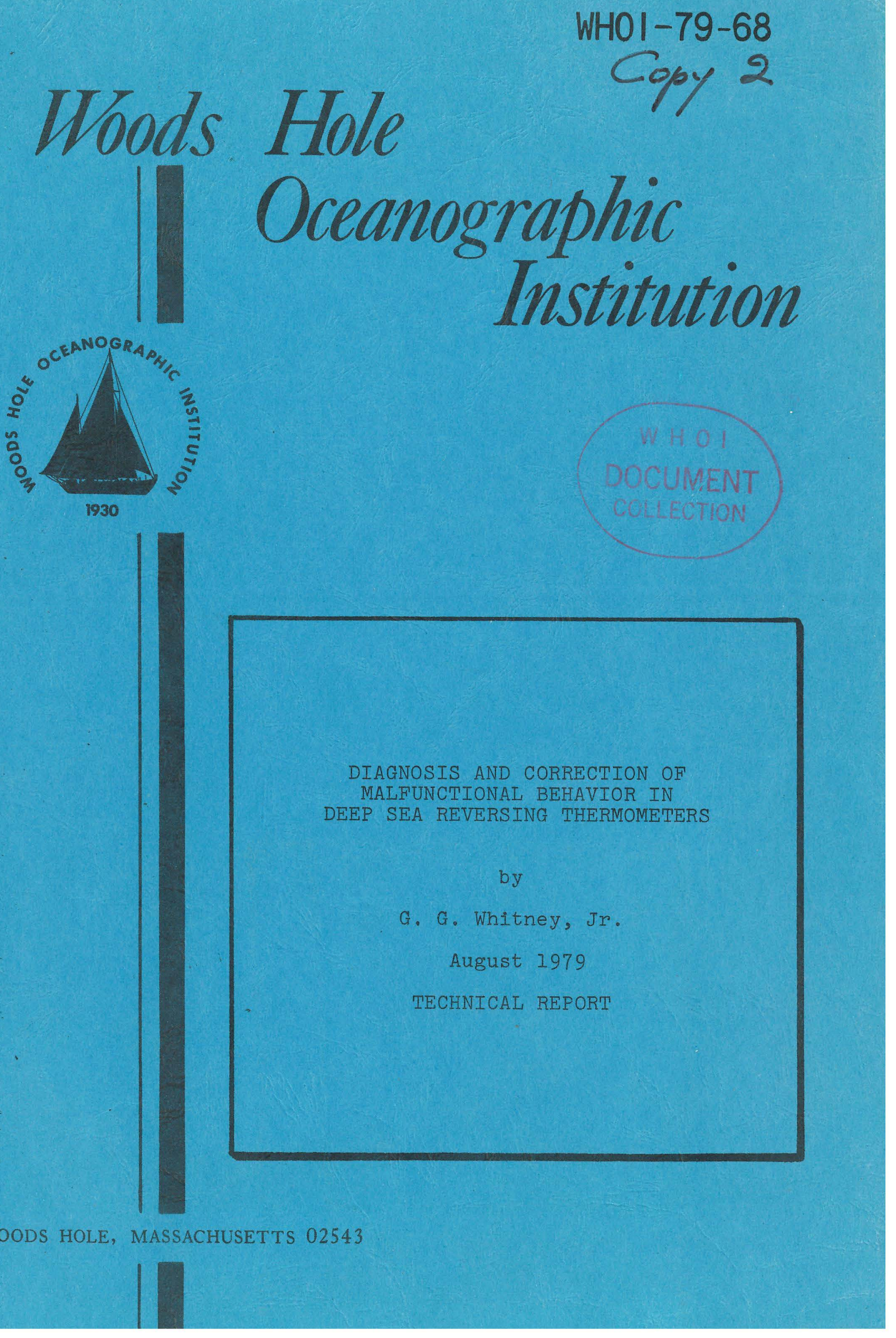


WHOI $-79-68$

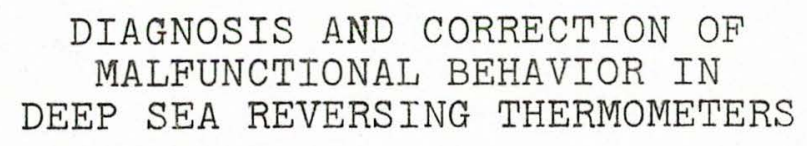

by

G. G. Whitney, Jr.

WOODS HOLE OCEANOGRAPHIC INSTITUTION

Woods Hole, Massachusetts 02543

August 1979

TECHNICAL REPORT

Prepared under the support of the Woods Hole Oceanographic Institution.

Reproduction in whole or in part is permitted for any pur. pose of the Unites States Govermment. This report should be cited as: Woods Hole Oceanographic Institution Technical Report WHOI-79-68.

Approved for Distribution

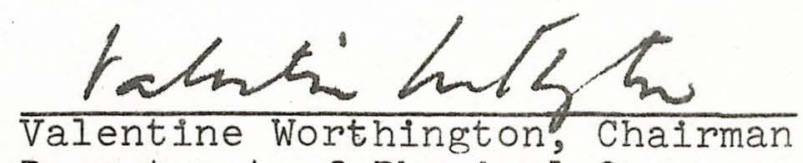

Department of Physical Oceanography 
i. Abstract ............... 2

I. Introduction ................ 2

II. Definitions. . . . . . . . . . . . . 2

III. Functioning. . . . . . . . . . . . 4

IV. Malfunctions .............. 5

V. Causes of Malfunctions . . . . . . . . . 6

VI. Manipulation for Removal of Bubbles. . . . . . 6

VII. Mercury Sticks in the Appendix... . . . . . . 10

VIII. Flooding................ 13

IX. Removal of Gas From Reservoir Protected DSRT's and Certain UDSRT's...... 14

X. Removal of Gas From Reservoir UDSRT's with Goose-neck Construction ...... 16

XI. Failure to Drain . . . . . . . . . . 17

XII. Mercury Separated in Throat of Bulb. . . . . . . . 19

XIII. Excessive Gas in DSRT System........... 21

XIV. Separated Auxiliary Mercury. . . . . . . . . 21

XV. Summary. . . . . . . . . . . . . 24

XVI. References and Acknowledgements. . . . . . . 25 


\section{Diagnosis and Correction of Malfunctional Behavior in Deep Sea Reversing Thermometers}

by G. G. Whitney, Jr.

\section{$\underline{\text { ABSTRACT }}$}

The nomenclature, morphology and functioning of deep sea reversing thermometers are identified and explained. The various types of malfunctions, nonfunctions and other aberrations are defined and detailed instructions are given for the procedures used to restore normal functioning to the extent possible. Advice is given regarding the use, handling, storage and transportation of these instruments so as to avoid damage and actions which might cause malfunctions to occur.

\section{INTRODUCTION}

In 1952, the author wrote a brief monograph, Technical Report No. 52-29 "Notes on Malfunctional Behavior and its Correction in Deep Sea Reversing Thermometers". This included, as an Appendix, a translation of the booklet from Richter \& Wiese, Berlin, "Uber die Funktion der Umkippthermometer sowie die Beseitigung von Storungen" ("Operation of the Reversing Thermometer - Richter - and the Elimination of Disturbances"). The text of T.R. No. 52-29 was incorporated in its entirety into U. S. Navy Hydrographic Office Publication $k^{6} 607,1955$ edition, and for many years was the accepted treatise on the subject. T.R. No. 52-29 has been out of print for a number of years and H.0. Publ. 非 607 has been revised several times since 1955, dropping the WHOI-R\&W text in the process. It seems appropriate at this time to revise and augment the 1952 monograph, restating the substance of the Richter and Wiese pamphlet and reflecting the observations made and knowledge acquired as the result of twenty-eight years of experience in calibrating and manipulating many thousands of reversing thermometers.

\section{DEFINITIONS}

To ensure clarity and understanding of the subject, the terms which are used in this dissertation are defined as follows:

Reversing thermometer (referred to herein as DSRT): a mercury-in-glass thermometer so designed and constructed that when it is rotated (reversed) $180^{\circ}$ from its original, vertical, sensing attitude or position to its reading position, a specific volume of mercury, representative of the temperature (or temperature and pressure) sensed by the instrument at the moment of reversal, is broken off from the remaining mercury and flows down to the bottom end of the instrument and comes to rest in a graduated stem to provide and preserve a temperature (or temperature plus pressure) reading.

Protected reversing thermometer (referred to herein as protected DSRT): a reversing thermometer completely sealed in a cylindrical glass shell so as to be unaffected by water pressure; it records temperature only. The she11 
is intended to be able to withstand the pressure of water at depths down to 7000 meters, or deeper if extra heavy glass is used in the shell. (Fig. 1 See also for other terms below.)

Unprotected reversing thermometer (UDSRT): a reversing thermometer in a cylindrical glass shell with one end open so that it is affected by water pressure as well as by temperature; it records temperature augmented by a pressure component. The pressure component is used to calculate the depth of the instrument below the sea surface at the moment of reversal.

Auxiliary thermometer: an ordinary mercury-in-glass thermometer placed within the glass shell of the DSRT to indicate the temperature inside the shell at the time of reading the "main" thermometer. Simple reversing thermometers without auxiliary thermometers are made for marine use where accuracy to greater than 0.1 to $0.2^{\circ} \mathrm{C}$ is not required.

Reservoir: the generally cylindrical, glass portion of the main thermometer which holds the principal body of mercury which senses the ambient temperature (and pressure in a UDSRT).

Bulb (sometimes called "small bulb"): the small expansion chamber at the opposite end of the main thermometer from the reservoir.

Stem: the straight, graduated portion of the thermometer on which the temperature reading appears after reversal.

Appendix: the small branch or "dead-arm" (from the German "toten Arm") off the main capillary channel near the reservoir. The mercury is supposed to break, on reversal, at the junction of the appendix with the capillary.

Pigtail: the $360^{\circ}$ turn, or equivalent thereof, of the capillary between the appendix and stem which acts as a trap for any mercury which may extrude beyond the appendix and break off as the result of a temperature rise after reversal.

Capillary: the bore of the thermometer from the reservoir to the bulb, in which the mercury moves. "Capillary system" includes the bulb.

Throat of the bulb: the point at which the straight, uniform capillary diameter of the stem begins to open out to form the bulb.

Throat of the reservoir: that portion of the capillary between the reservoir and the appendix.

Mercury: In this discussion, the word "mercury" alone always refers to the internal mercury of the main thermometer; other mercury will be specifically identified.

Mercury bath: the loose mercury surrounding the reservoir of a protected DSRT. This body of mercury is in no way involved in the functioning of the thermometer other than in transmitting heat to or from the mercury in the reservoir. 
Plug: the stopper, generally of cork, in a protected DSRT which holds the main thermometer and attachments in place inside the shell and retains the mercury bath in the reservoir end of the shell.

Correct breaking point, Break-off point: the one and only point at which the mercury is supposed always to break at reversal. This is the junction of the appendix with the main capillary channel. (Fig.2).

Upright: that vertical position of a DSRT in which the reservoir is down. To "Right" is to move the instrument to the upright position. This is the "sensing position".

Reversed: that vertical position of a DSRT in which the reservoir is up. This is the "reading position". To "Reverse" is to move the instrument to the reversed position.

Drain: the flowing back of the mercury from the bulb upon righting a DSRT after reversal to rejoin the principal body of mercury in the reservoir and contiguous capil1ary.

Bubble: a small quantity of gas (air and mercury vapor) within the mercury column which may cause a false separation of the mercury (malfunction) at its locus when the thermometer is reversed. A bubble may or may not be visible, depending upon its size, location within the capillary and the spacial attitude of the thermometer.

Flood: the filling of the entire capillary system of the main thermometer with mercury, at or after reversal, with no separation of mercury, leaving a large, visible bubble (void) in the reservoir. This is a malfunction and is discussed below.

Manipulation: the techniques used to correct malfunctions and to attempt to restore normal functioning of a DSRT.

\section{FUNCTIONING}

In normal function, when a reversing thermometer is moved from the upright to the reversed position the main mercury column should start to move out of the appendix and toward the bulb about the time that the instrument has reached a horizontal position. This mercury-in-motion is essentially the mechanism by which the molecular cohesion of the mercury in the capillary is abruptly broken or torn apart as the last of the mercury initially in the appendix clears the appendix and thus separates from the remaining mercury in the throat of the reservoir. This remaining mercury is effectively "anchored" by its cohesiveness with the mass of immobile mercury in the reservoir itself.

The volume of the mercury thus separated at reversal is representative of the temperature (or temperature plus pressure in a UDSRT) sensed by the mercury in the reservoir at the moment of reversal. It is intended and expected to flow down into and completely fill the bulb, with part of its total volume standing up into the stem, where a reading may be 
made by an observer. The main capillary system is almost totally evacuated of air, so that only a trace of gas (air and mercury vapor) exists internal1y. This permits the mercury to run freely from the appendix into the bulb and back again as the attitude of the thermometer is changed. Too much gas will cause problems which will be discussed below; too great an internal vacuum is suggested by some manufacturers to be a cause of difficulty in draining after reversal.

\section{MALFUNCTIONS}

For a variety of reasons, DSRT's do not, unfortunately, always function in the proper manner. The types of malfunctions most frequently encountered will be described below and later the various methods of correcting them will be discussed. To determine what sort of malfunction has occurred, the instrument must be examined carefully with a reading glass, generally a loupe of about 5X magnification. In all the various manipulations and procedures described in this discussion such glass should be used at all times to observe the mercury and the progress of the manipulations.

1. Upon reversal, the mercury separates at some point other than the correct breaking point. If the separation occurs in the throat of the reservoir, the mercury will have run out of the appendix, leaving the appendix clear. The result of such a malfunction will be a reading too high. If the separation occurs beyond the appendix (toward or in the pigtail) the mercury behind the point of separation will generally not have moved at all and the appendix will remain full. The result in this case will be a reading too low. (It is possible that with a bubble beyond the appendix the mercury in the appendix and between the point of separation may have moved slightly, leaving the appendix partly empty.)

2. Upon reversal, the mercury separates in the throat of the reservoir and some or all of the mercury initially in the appendix remains there. The result will generally be a reading too high. A variation of this is the case in which the mercury breaks at the correct breaking point, but leaves the appendix still filled with mercury. The result of this would be a reading too low by the volume of mercury retained in the appendix.

3. Upon reversal, the thermometer floods: that is, no separation of mercury occurs. Actually, flooding may take place at any time after reversal and it is impossible to determine at what point in time it occurred. This will be discussed below.

4. Upon reversal, the mercury does not flow or separate at a11, unless or until the instrument has been jarred or tapped, perhaps quite solidly, by which time the temperature may have changed significantly. This action will frequently cause the instrument to malfunction in one of the ways described above.

5. Not really a malfunction, since the DSRT has behaved normally at reversal and provided a valid reading, is the failure of the mercury to drain upon righting without recourse to tapping, jarring, shaking or other more drastic measures. 
V. CAUSES OF MALFUNCTIONS

The most common cause of malfunction in a reversing thermometer is the presence of a bubble of gas within the mercury column somewhere in the capillary system. Normally, whatever gas is present in the thermometer is above the mercury column when the instrument is in an upright position. On occasion, however, generally as the result of vibration or rough handling of the instrument in its reversed position, such gas, or part of it, moves up through the mercury column, and wherever it settles, will frequently cause a separation of the mercury at that point instead of at the break-off point when the thermometer is again reversed. Such bubbles can result from leaving the DSRT's in the storage or reading racks at sea in the reversed position for extended periods. When, after a cast at sea, the DSRT's have been read and the readings recorded, the bottles, with their DSRT's should be returned to the upright position. This must be done in any case prior to the succeeding cast and it is best to do it at once so that the DSRT's, subject to vibration, do not remain in the reversed position any longer than necessary. DSRT's should never be transported or stored in the reversed position except under the unique condition of extreme cold, which may cause serious problems in the auxiliaries. (See Section XIV.)

On occasion, with the presence of a bubble in the capillary ahead of (below) the appendix, the mercury may separate at the bubble and also run out of the appendix and separate at the break-off point. In such case, the mercury separated beyond the bubble will run down into the bulb, possibly extending up into the stem, giving a false, low reading, while the balance of the separated mercury, that between the bubble and the break-off point, will fall into the pigtail and be held there. This is of no import, as the middle section of mercury (in the pigtail) will be picked up in the course of the manipulation sequence described below.

VI. MANIPULATION FOR REMOVAL OF BUBBLES

Every faulty separation of the mercury must be presumed to be due to a bubble until some other cause can be established and the fundamental manipulation procedure should always be used first. When a faulty separation has occurred, the bubble responsible must be moved forward (upward) in the capillary into the end of the bulb where it belongs. Manipulation of protected DSRT's are necessarily performed with the thermometer in the she11; UDSRT's can and should be removed from the she1ls for manipulation. The procedure and sequence of operations are as follows:

A. With the mercury improperly separated, presumably the result of a bubble, hold the DSRT essentially horizontal at the point of balance in the hand and heat the reservoir, using a moderate flame from a gas or alcohol burner so that the mercury in the reservoir will expand and move forward in the capillary, pushing the bubble ahead of it. This heating must be done carefully, constantly turning the thermometer about its central axis and moving it in and out of the flame so that the heating will be uniform and not too rapid, otherwise it is possible to crack or even break the protected shell or the glass structure (reservoir or part of the capillary) of a UDSRT. In addition to possible breakage of glass and ruining the thermometer, the release of hot mexcury into the atmosphere is highly dangerous, as mercury vapor is extremely poisonous, and even in its normal liquid state mercury is an unpleasant, hazardous substance to have loose in the laboratory. 
Depending upon where in the capillary the original false separation occurred and on the internal volume of the pigtail, this first heating should move the forward end of the mercury column to: (a) half-way around the first pigtail turn $\left(90^{\circ}\right)$; (b) half-way around the second pigtail turn $\left(270^{\circ}\right)$; (c) all the way around and through the pigtail to a point just short of the stem near the metal fitting retaining the auxiliary thermometer. (These points are shown in Fig. 3) At this stage, the mercury should not be made or allowed to enter the stem itself unless it can be determined that the bulb is not full of mercury. The criterion for which approximate stopping point to use for forward progress of the mercury in the capillary is how hot the reservoir or the reservoir end of a protected shell has become to the touch. As long as the heated glass can be brushed lightly across the fingers or palm of the hand without noticeable pain it is presumably not too hot. In the absence of a flame, running hot water from a hose or faucet or hot air from a heat gun may be used. CAUTION - In all these heating procedures, the progress of the mercury in the capillary must be watched, to be sure that the expanding mercury does not go so far as to meet the separated mercury, filling the entire system, since the internal pressure thus developed in the system as the result of any further heating and expansion of the mercury will almost certainly break the reservoir, ruining the instrument. It should be noted that because of the relatively large bore of the capillary in the pigtail, the mercury will advance quite slowly as it expands with heating. However, should it be permitted to enter the very tiny capillary of the main stem, it will move extremely rapidly as it expands further.

If the separation occurred back in the throat of the reservoir, the first heating may not be able to cause the mercury to expand to point (a) before the glass gets too hot. In such case, heating should be stopped after the expanding mercury has passed the appendix and is at the first bend in the capillary. In rare instances, where mercury separation has occurred far back, almost at the reservoir, in a protected DSRT the first heating may have to be done "blind", as the plug will obscure the end of the mercury column. Do not overheat.

B. When sufficient heat has been applied to move the forward end of the mercury column to one of the preferred positions noted above, right the DSRT and allow the mercury in the bulb and far end of the stem to flow down and rejoin the rest of the mercury at the new locus of the bubble. Now cool the reservoir, slowly at first by blowing on it, wiping it on a towel or other material and ultimately chilling it in water, ideally ice water, for perhaps one minute. This cooling will cause the mercury to contract into the reservoir. At the earlier rejoining of the two bodies of mercury, the bubble should have been pushed out to the capillary wall, where it should remain, in situ, while the contracting mercury flows down past it. Note that putting hot glass directly into ice water might break the shell or thermometer.

C. With the reservoir now chilled, grasp the upright DSRT in the full palm of the left hand, with the pigtail in the center of the palm and the axis of the pigtail helix an extension of the axis of the extended forearm and wrist and rotate the DSRT smartly inward about the forearm axis, with a snap, through $90^{\circ}$ to the horizontal position. This rapid motion 
should cause the mercury column to separate again at the bubble in its new, second location. Using a reading glass, verify this separation at the expected point (at the bubble locus) and if it did occur as desired, incline the instrument further toward the reversed position so that the separated portion of mercury will run toward the bulb. This mercury should be observed carefully as it enters the bulb to determine 1) if it is going to fill the bulb completely, with some mercury standing up into the stem to at least near the $0.0^{\circ}$ mark or 2 ) if its volume is small enough so as not to fill the bulb completely, leaving a definite void in the bulb when all of this body of mercury has been moved to the far end of the bulb. If either of these two conditions will exist, the reversing motion may be completed. In the first instance, now perform a second heating cycle, moving the bubble to point (c) as in " $\mathrm{A}$ " above, followed by righting the instrument, rejoining the mercury, cooling again and snapping the DSRT through a $90^{\circ}$ arc as described in " $B$ " and in " $C$ " above. In the second instance, after the first cooling and forcible separation of the mercury at the bubble, the end of the mercury column ought to be already at or near point (c) described in " $A$ " above.

The purpose of rotating the DSRT rapidly $90^{\circ}$ from upright to horizontal about an axis passing through the center of the pigtail helix is to use the centrifugal force of such motion and the inertia of the mercury beyond the bubble to assist this mercury to move toward the bulb while restraining the mercury behind the bubble from moving out of the appendix or at a11. The reason for the use of the left hand for this motion, is that the natural, wrist-arm inward rotation is clockwise for the left hand and counter-clockwise for the right. Since the pigtails of all DSRT's are routinely of left hand (counter-clockwise) helical construction, the above-described snap rotation should be clockwise to generate the desired motion and effect. Such clockwise motion can, of course, be generated with the right hand but it is a little awkward, apt to be not as rapid and in the particular situation to be described in the next paragraph makes the rapid shifting of the DSRT from the snap rotation grip to one in which the DSRT is held horizontal at the mid-point in the right hand with the fingers down very awkward.

If a bubble should at any time be located in a straight portion of the capillary which is essentially parallel to the central axis of the thermometer, such as between the reservoir and the pigtail or just before the beginning of the stem, instead of the above snap wrist movement the proper motion should be one in which the $90^{\circ}$ rotation is performed while the entire instrument and arm is being moved suddenly downward and outward to the side through a $90^{\circ}$ arc of roughly $15-18^{\prime \prime}$ radius. In such situation, this maneuver may be performed with either hand.

Many low-range $\left(3^{\circ}, 6^{\circ}, 8^{\circ}\right)$ DSRT's have a pigtail in which the major part of the total internal volume is in the first $180^{\circ}$ turn nearest the beginning of the stem and this portion will generally have a very large sectional area. Because of the fine capillary diameter in the stem, the mercury will move only slowly in such instruments. In the manipulation sequence, if the bubble, after a heating-cooling cycle is at point (a) near the point of greatest sectional area of the pigtail, after the $90^{\circ}$ snap rotation to the horizontal position, instantly transfer the 
DSRT from the left hand (at the pigtail) to the right hand at about the mid-point of the instrument, with the first turn of the pigtail nearest the manipulator (DSRT horizontal) and strike the thermometer from below, at the pigtail, several times rapidly with or against a knuckle of the left hand. With a large sectional area of the pigtail at and beyond point (a) such tapping will generally cause the slowly expanding bubble to move upward around the first $180^{\circ}$ pigtail turn to where the sectional area suddenly becomes much smaller, at which point total separation of the mercury beyond the bubble will occur. The result is to move the bubble a substantial distance through (around) the pigtail without an additional heating-cooling cycle and with the bubble now not far (in internal volume) from point (b); subsequent heating will now move the bubble through points ( $b$ ) and (c) and presumably through the stem into the throat of the bulb.

D. When the manipulation sequence has reached the stage where there is or is going to be a definite void in the bulb and the bubble is presumably at about point (c), if the now relatively small volume of mercury beyond the bubble does not run down and fall into the end of the bulb with the DSRT in the reversed position, hold the instrument firmly in the hand by the reservoir end and give it a relatively smart snap downward from horizontal to the reversed position, as one might a clinical thermometer; the centrifugal force of this motion will drive the separated portion of mercury into the end of the bulb. (Fig. 6.) Now heat the reservoir again so that the principal body of mercury expands along and preferably through the stem and into the beginning of the bulb. Reverse the thermometer; the mercury should flow out of the appendix and run down into the bulb in the normal manner. As it rejoins the mercury already in the bulb, pushing the bubble ahead of it, the bubble will be pushed out to the wall of the bulb, where it will frequently be visible even to the naked eye. (Fig. 4.)

E. This bubble in the bulb must now be moved out to the end of the bulb, beyond and above all of the mercury in the capillary. The preferred, easiest and quickest method is as follows: Hold the DSRT, partially righted, perhaps 40-45 from horizontal and move it downward along its axis (toward the reservoir end) rather smartly and stop it suddenly with a slight, upward, return motion, rather as one might shake ketchup out of a bottle. This motion should be carried out with the thermometer held firmly in the fingers of both hands, one hand near the reservoir, one near the bulb end; with only one hand, there is risk of losing control of the instrument and dropping it, with disastrous results. The effect of this motion is to hold the mercury in the end of the bulb in place while inducing expansion of the bubble and downward movement of the mercury below the bubble; the moving mercury, being molecularly cohesive, will tend to flow downward around the expanding bubble, pulling with it more mercury from above as the expanding bubble moves upward in the bulb until a new void is created further toward the end of the bulb. (Fig. 5.) When total separation of mercury in the bulb has occurred (void between the two bodies of mercury) incline the instrument again about $45^{\circ}$ toward the reversed position and rejoin the free-floating body of mercury with that remaining in the end of the bulb. Successive repetitions of these motions (incline toward upright-shakeincline toward reversed-rejoin) should ultimately move the bubble out to the end of the bulb, above all the mercury, where it is supposed to be. 
In the case of relatively low range DSRT's $\left(6^{\circ}, 8^{\circ}, 10^{\circ}\right.$, or $15^{\circ}$ ) the inside diameter of the stem portion of the capillary is relatively narrow and the bulb relatively large in sectional area except near the throat and the mercury moves in and out of the bulb slowly. Under such conditions, as the bubble in the bulb begins to expand as described above, return the DSRT closer to a horizontal position, to roughly $20^{\circ}$, hold the instrument firmly in one hand by the reservoir end and strike the bulb end downward against a knuckle of the other hand a number of times in succession. The expanding bubble should move upward in the bulb with each tap until it is above all the mercury. The degree of expansion of the bubble permitted, so as to prevent formation of a void across the total diameter of the bulb until it has reached the end, can be controlled by varying the degree of tilt of the DSRT in this process. This is essentially the same action described in the third paragraph of "C" above.

In some cases, particularly if the bulb is relatively long and thin or if there is a tendency for the mercury to stick in the bulb tip, the techniques described in the two previous paragraphs are not effective and the classic Richter and Wiese method should be used. Hold the DSRT firmly in one hand near the reservoir, essentially horizontal. Strike the bulb end of the DSRT with some sharpness against a knuckle of the other hand so as to dislodge one or more globules of loose mercury from the portion of mercury in the end of the bulb. Now partially reverse the thermometer so that the main body of mercury will flow down into the bulb; do not allow this to fill the bulb but allow it to advance only far enough to pick up the separated loose mercury globules; return the instrument to a more nearly upright position so that the main body of mercury exits the bulb or at least retreats as far as it can toward the reservoir end. Repeat this cycle of knocking off mercury globules and picking them up with the main body of mercury until all mercury in the bulb has been rejoined to the main body and all gas is above it in the end of the bulb. In performing this operation, great care must be taken not to strike the DSRT too hard against the free hand, as it is possible thus to cause the heavy (auxiliary reservoir) end of the auxiliary thermometer to break off at the metal mounting fitting.

\section{MERCURY STICKS IN THE APPENDIX}

Al1 too frequently, malfunctions occur because the mercury does not flow out of the appendix at reversal as it is intended to do. There are various causes for this condition. (Fig. 7.)

1. There may be impurities in the mercury which have a chemical affinity for the glass or for impurities in the glass.

2. Strain cracks, resulting from improper annealing or normalizing of the thermometer glass during manufacture may appear with age. Mercury molecules may tend to get caught in these, effectively anchoring the mercury to the appendix wall or tip.

3. Minute internal fractures may be produced in the appendix tip by striking the DSRT from below against a hard object while in the upright position with the weight of the mercury impinging on the appendix. Cautions on this subject will be expanded upon later in this discussion.

If a faulty separation of the mercury has occurred at reversal, even if the appendix remains full of mercury, it must first be assumed that 
the cause may be a simple bubble and the manipulations described in the preceding section should be carried out at least once and preferably three or four more times if the first manipulation sequence is not effective. When it becomes apparent that the mercury will not flow out of the appendix without lateral jarring of the instrument or tapping from below (reversed) a defect in the appendix can be assumed. The severity of the condition can be divided into perhaps three categories.

A. If a light, lateral tap of the instrument in a reversed position causes the mercury to flow out of the appendix and break off in a normal manner, the DSRT is probably useable at sea, at least with Nansen bottles. (This will be explained below.) This condition might be described as "slightly sticky".

B. If a firm to strong lateral tap of the reversed DSRT is required to cause the mercury to move out of the appendix and separate at the break-off point, the DSRT is definitely "sticky" and in need of repair before it can be used with any confidence at sea. A DSRT in such a condition is unlikely to receive an adequate jolt to induce functioning when reversed at sea. It will either not function at all (or malfunction) or the mercury will flow out of the appendix and break off at some later time, when the instrument is sensing a temperature and/or pressure different from that at the time of reversal. This might occur during retrieval as the result of wire vibration or contact of the bottle with the side of the ship or jarring during removal of the bottle from the wire and placing it in the reading rack. The result will be either no reading (no separation) or a false reading (malfunction or late function).

C. If, with tapping of any sort or even without tapping, the mercury persistently (not necessarily always) breaks at some random, varying place, above or below the appendix, leaving mercury in the appendix after reversal, the DSRT is seriously defective and of no use unless and until repaired by a competent DSRT glass artisan.

In almost all instances, from that in which the DSRT is only slightly sticky to the extreme of the mercury being firmly stuck in the appendix, if the appendix can be cleared of all the mercury which can be made to flow out of it, examination with a reading glass, $5 \mathrm{X}$ or stronger, will reveal a speck (or specks) or even perhaps a significant quantity of mercury which seems to be habitually resident in the appendix, most commonly at the tip, or minute fractures in the glass observable with certain light angles. In conducting such an examination, one may note that optical distortion of the appendix image by the glass structure at certain angles or with certain lighting may make shadows or dark spots which may seem to be mercury but are not. Rotate the DSRT about its axis, observing the appendix area from all sides and with the light coming from various angles. Light should be essentially behind the thermometer, transilluminating the appendix area, and the observations should be made both with an indirect light source such as a well-lighted white wall, and against a direct light source, preferably fluorescent.

If the mercury will not flow out of the appendix after a firm lateral jolt, as might be applied with the reading glass, a firm tap may be given from below (DSRT reversed). If the mercury still will not exit the appendix, grasp the DSRT horizontally in the full palm of the hand, holding 
it loosely but not so 1 oosely that it can fall out laterally, and strike the bulb end axially against a solid wooden surface. The sudden stop in this maneuver should cause the momentum of the mercury to "pop" the mercury out of the appendix. If this maneuver is not successful with the DSRT horizontal, it may be tried with the instrument vertical (reversed). The amount of force permissible cannot be accurately described and one must use one's own best judgment as to when the energy threshold may reach the point where breakage may result. If in doubt, further attempts to clear the appendix by such forceful methods should be abandoned and the instrument set aside for repair. It is possible with such maneuver to cause the thermometer assembly in a protected DSRT to move axially within the plug or to unseat a poorly cemented plug, dumping the bath mercury into the body of the she11, which would make repair necessary in any case. In the case of a UDSRT out of its she11, such maneuvers must be rather less violent, and in the situation where the auxiliary reservoir extends beyond the tip of the main bulb, such axial striking cannot be used without danger of breaking the auxiliary reservoir. One could conceivably replace the UDSRT in its shell and try the maneuver in such condition but the point contact of the metal mounting lugs with the mounting holes in the shell might very possibly cause breakage of the shell when such a sharp blow was given.

It should be noted that in all of these various maneuvers to dislodge mercury from the appendix, each time a jar or blow is given, if the mercury does not leave the appendix, it will almost certainly separate somewhere. Before the next shock is given, all the mercury must again be rejoined as a continuous mass, otherwise such jarring to empty the appendix cannot be effective.

With a UDSRT out of its she11, it is possible, using a small flame and great care, to heat the appendix area of the capillary of a UDSRT to the point where any mercury remaining there will be vaporized (about $360^{\circ} \mathrm{C}$ ) leaving the appendix temporarily free of visible mercury. Care must be taken not to overheat the reservoir in this process and after the mercury has vaporized, the hot glass must be allowed to cool slowly, without any inducement, until it is no longer hot to the touch. This is a potentially dangerous maneuver, from the risk of glass breakage and possible vaporization or spillage of loose mercury, and in most cases does not cure the condition because there may still be remaining invisible molecules of mercury, amalgamated to impurities, which will unite with the main body of mercury when it again fills the appendix. In addition, if the problem is the result of glass fractures, the glass temperature attained in this process is not nearly high enough to eliminate these. This would require the expertise of a competent DSRT glass artisan, who should be able to achieve a temperature at which the glass would be slightly plastic and the fissures close, probably altering slightly the shape and/or volume of the appendix in the process.

Earlier in this section, reference was made to potentially successful use of slightly sticky DSRT's in Nansen bottles. Such DSRT's are apt to work satisfactorally in Nansen bottles, since the reversing action or motion of a Nansen bottle is such that the modest centrifugal force acting on the mercury during reversal is all in one direction and assists the mercury to flow and exit the appendix. In addition, reversal of the Nansen 
bottle is apt to be accompanied by a certain amount of shaking or vibration and the snap of the valve connecting rod locking spring is apt to impart a slight shock to the DSRT's carried by the bottle, any and all of which would assist initiation of mercury movement out of the appendix at reversal. By contrast, the reversing motion of a simple, spring loaded DSRT reversing frame and that of the reversing mechanism of a Niskin bottle is about a point near the middle of the DSRT's, so that while the centrifugal force in reversal is helping part of the mercury, from near the middle of the stem to the bulb, to flow toward the bulb, such force acts in the opposite direction on the mercury between mid-stem and the tip of the appendix, inhibiting movement of the mercury out of the appendix at reversal. The action of the simple reversing frame is relatively gentle and the frame ends up against a solid stop where it latches, so that the jar of stopping should encourage flow out of the appendix at the end of the reversing motion and assist proper function of the DSRT. Reversing motion of the Niskin mechanism, however, is relatively rapid and violent, with initial quick acceleration and much more centrifugal force and without any terminal jar against a stop. There are indications that the Niskin mechanisms sometimes cause malfunctions in perfectly normal DSRT's as the result of centrifugal force acting in both directions along the thermometer axis and tearing the mercury column apart at some point near the middle of the capillary.

VIII. FLOODING

Flooding is caused by the presence of gas in the reservoir. If there is gas in the reservoir, a DSRT may flood, occasionally, frequently or consistently. This flooding may take place at the time of reversal, during retrieval of the cast or later in the reading rack. A DSRT which is also sticky will flood more often than one which is not. If a DSRT is retrieved flooded, there is no way of determining whether such flooding occurred at the time of reversal or during retrieval as the result of vibration on the way up or of striking the side of the ship. The greater the increase between the temperature at the time of reversal and that at the time of a late flooding, the more likely flooding is to occur (if there is gas in the reservoir). This is because with an increase in temperature after reversal, expansion of the mercury causes it to extrude beyond the break-off point and into the pigtail; as the volume, and hence weight, of this mercury increases with rising temperature, it develops more and more "lead" into the pigtail and at some point, if the DSRT is jarred slightly, this may be enough to start a flow of mercury out of the reservoir. With a normal DSRT, having no gas in the reservoir, when a certain amount (weight) of mercury had been extruded beyond the break-off point, it may be expected to break away, at the break-off point, as the result of its own weight (or a jar) and fall into the pigtail where it should be trapped and held. Occasionally, DSRT's may be accused of "flooding" as the result of relatively large temperature rises above the reversal temperature (up to $40^{\circ} \mathrm{C}$ ). This is not truly flooding in the sense used in this discussion, since there is no gas and no void in the reservoir. This falling through of the mercury extruded beyond the break-off point as the result of large temperature rise is caused by a structural deficiency in the thermometer itself, in that the pigtail does not have sufficient volume (capacity) to 
trap and hold $40^{\circ} \mathrm{C}$ of mercury as required by standard DSRT specifications. In rare cases, the pigtail capacity may be as little as $30^{\circ} \mathrm{C}$. Such a falling through of mercury could presumably only occur in a protected DSRT, since the mercury in a UDSRT would have shown a reading usually substantially higher than the temperature of reversal, due to the pressure component, and when brought to the surface, the mercury would have retreated a considerable distance behind the break-off point so that a temperature rise substantially higher than $40^{\circ} \mathrm{C}$ would have to be experienced for the expanding mercury to fill the pigtail and fall through. To determine if there is gas in the reservoir, proceed as follows: A. Chill the reservoir, in ice water, if available, then reverse the thermometer; a temperature of less than $10^{\circ} \mathrm{C}$ is desirable, less than $5^{\circ} \mathrm{C}$ preferred.

B. With the DSRT still reversed (vertical) heat the reservoir to a temperature perhaps $35-40^{\circ} \mathrm{C}$ higher than that of reversal (no precision required). Mercury will expand out of the reservoir into the pigtail. In a normal instrument, at some time during the heating, this mercury may separate, at the break-off point, from its own weight, and run down into the pigtail. If the pigtail is capable of holding up to $40^{\circ} \mathrm{C}$ volume of mercury, as it should be, and the heating has not raised the temperature more than $40^{\circ}$ higher than that of reversal, such separated body of mercury will be trapped in the pigtail.

C. After heating as above, if the extruded mercury has not broken off of its own accord, shake the thermometer axially downward to cause separation. If separation occurs at the break-off point, either with or without shaking, there is no gas in the reservoir.

D. If there is gas in the reservoir, no separation will occur under the conditions of either $B$ or $C$ above and mercury will flow down from the reservoir, filling the entire capillary system and leaving the tell-tale bubble in the reservoir.

E. It is entirely possible that there may be gas in the reservoir of a DSRT which is also somewhat "sticky", so that the light tap needed to cause the mercury to leave the appendix may also be enough to start the main body of mercury moving out of the reservoir. In such case, if one can eliminate the gas from the reservoir, the instrument may then be capable of use, at least in Nansen bottles. (See Section VII.)

IX. REMOVAL OF GAS FROM RESERVOIR - PROTECTED DSRT'S AND CERTAIN UDSRT'S

Gas in the reservoir of a protected DSRT and of UDSRT's which have the reservoir extending straight along the thermometer axis as in protected DSRT's (Negretti \& Zambra and $\mathrm{K}$. Gohla) instead of the more common, unprotected "goose-neck" construction can usually be removed in the following manner:

A. Line the inside of a $1000 \mathrm{cc}$ beaker or comparable vessel with a strip of $1 / 4^{\prime \prime}$ foam rubber or similar substance perhaps $3^{\prime \prime}$ wide; if the container is of glass, a metal disc of appropriate size should be placed on the bottom to avoid contact of the thermometer or its shell with the glass of the vessel. Place several pieces of dry ice $\left(\mathrm{CO}_{2}\right)$ in the vessel thus prepared and partially fill the container with ethyl alcohol (or any other liquid which will not freeze at the dry ice temperature). It is helpful if 
the container is set on and wrapped with some insulating material. Another bath of icewater should also be prepared.

B. Reverse the thermometer at any (room) temperature, but bring it back to a horizontal position before the mercury has entirely filled the bulb, leaving perhaps one-fifth or less of the bulb's volume empty; there must be some void in the bulb beyond its throat, otherwise one may create a condition where the bulb is full to the throat and the mercury tends to stick in the bulb. Hold the thermometer firmly near the reservoir end and snap it sharply downward as one might a clinical thermometer, returning it instantly to a horizontal position. The object of this is to cause the mercury to separate in the throat of the bulb, so that a large mass of mercury is driven to the outer end of the bulb, leaving a small void at the inner end of the bulb near the throat. (Fig. 6) This mass of mercury will generally hang or stick in the outer end of the bulb when the instrument is carefully righted; if it will not do so, but runs down into the stem to rejoin the main body of mercury, the method cannot be used.

C. There is now a substantial volume of mercury "stored" in the end of the bulb and another mass loose in the capillary between the bulb throat and the break-off point. Hold the middle of the DSRT firmly in the full hand, in a horizontal position, and execute a smart $180^{\circ}$ twist of the wrist; this will hold the separated mercury in the bulb and drive the other portion of loose mercury back into the pigtail area. At this point, use judicious shaking or twisting of the DSRT to get this loose mercury as far back in the pigtail as it will go without too much effort.

D. Heat the reservoir as necessary to rejoin the mercury extending from the reservoir with that now in the pigtail, then cool the reservoir as necessary until the pigtail is perhaps one-half to two-thirds full of mercury.

E. Hold the DSRT horizontal or inclined slightly toward the reversed position at its balance point loosely in one hand and strike the bulb end axially with the palm of the other hand; the DSRT should move an inch or so in the holding hand and inertia should cause the mercury to move out of the appendix and separate at the break-off point. Now shake the thermometer axially, using both hands, holding it somewhat inclined toward the reversed position so as to move the mercury now loose in the pigtail around the pigtail well into the second turn so that it will not run back into the appendix and throat of the reservoir when the thermometer is again upright. Right the thermometer. There is now mercury stored in the bulb and more stored in the pigtail. The appendix must be empty, and the reservoir is probably near room temperature.

F. With the DSRT now upright, mercury stored in the bulb and in the pigtail, appendix clear, chill it first in ice water, wipe off excess and dip it into the super-cold alcohol bath; after a couple of dips it may be left in the bath. Move the DSRT around in the bath with a circular motion to get more rapid and uniform chilling and also from side to side, striking it against the now rigid (due to cold) rubber lining; the thermometer may also be tapped gently vertically against the metal bottom plate of the vessel. This must be done delicately and judiciously so as not to cause either of the two stored bodies of mercury to move downward; if this were to happen, the entire sequence would have to be started again from 
the beginning. The tapping, against the vessel sides and bottom is intended to ensure that any gas in the reservoir moves upward toward the throat so that it will be above all the mercury in the reservoir. What is now happening is that with the extreme cold, all the mercury not stored in the bulb or pigtail is contracting entirely out of the throat of the reservoir into the reservoir body itself, ultimately leaving a void in the upper end of the reservoir into which any gas in the reservoir is expected to move. CAUTION: This chilling in the alcohol bath takes only a minute or a little more; the thermometer must be withdrawn from the bath frequently to note when the bath mercury of a protected DSRT begins to solidify (freeze) on the inner wall of the shell; as soon as this is noted, the super-chilling must be stopped, as it is dangerous to chill the reservoir so that the mercury inside solidifies totally.

G. With the DSRT now removed from the alcohol bath, still upright, allow it to warm; warming may be assisted by wiping with the hand, towel or such, but no direct heat should be applied until after the thermometer has warmed above $0 .{ }^{\circ} \mathrm{C}$. Lateral tapping may be continued until it can be seen that the mercury has expanded enough to have re-entered the throat of the reservoir. With the mercury moving up in the throat of the reservoir, one may now proceed with the heating and cooling sequences described in section VI for removal of bubbles from within the mercury column.

NOTE: in using this technique on a UDSRT with straight reservoir configuration ( $N \& Z$ or Gohla) the bare reservoir can only be left in the super-cooled alcohol for a relatively few seconds, and under no circumstances should the reservoir body be totally immersed in the bath; if this were to be done, the mercury in the throat of the reservoir might solidify, which would prevent all the mercury from being contracted into the reservoir and after removal from the super-cold bath, this frozen plug of mercury might not liquify before the warming mercury in the reservoir reached it, which would result in internal reservoir pressure and almost certain breakage.

X. REMOVAL OF GAS FROM RESERVOIR - UDSRT'S WITH GOOSE-NECK CONSTRUCTION UDSRT's having the common "goose-neck" construction of the reservoir are treated somewhat differently for removal of gas in the reservoir. If gas is present in a "goose-neck" reservoir, while the UDSRT is in the upright position it will normally be in the extreme end of the reservoir, opposite to the entry into the throat. Upon reversal, this gas will frequently expand in situ, leaving the bubble in the now bottom end of the reservoir, as reversed, unless the instrument has been jarred, causing the bubble to move to the throat end of the reservoir.

A. If the bubble (which will be visible) in the reservoir of a flooded UDSRT (goose-neck type) is still in the far end of the reservoir, tap the reservoir end laterally against a knuckle to induce the bubble to move up to the throat end.

B. Now chill the reservoir by holding it, still reversed, in a handfull of crushed ice so as to cause the body of mercury in the reservoir to contract.

C. Partially right the thermometer to roughly $10^{\circ}$ from horizontal so that the mercury in the throat and contiguous capillary begins to 
flow back (drain) into the reservoir. It may be necessary to shake or tap the UDSRT axially to initiate the draining but the bubble, now in the throat end, must not be permitted to move back toward the far end of the reservoir. The bubble will begin to shrink in volume, but must be retained in its locus at the entry to the throat.

D. When the bubble volume has been reduced by about half or more, return the thermometer very suddenly to the fully reversed position, coincidentally giving the reservoir end a smart, lateral tap against a knuckle. This action should cause the bubble to move up into the beginning of the throat, creating a separation of the mercury column at that point; the separated mercury will run down in the capillary towards the bulb, leaving the reservoir full of mercury, or nearly so, and a void (where the gas now is) at the beginning of the throat.

E. With the thermometer still reversed, heat the reservoir as much as seems prudent (see comment in Section VI) causing the mercury to expand up into the throat.

F. Now partially right the thermometer (roughly $20^{\circ}$ from horizontal) giving it an axial tap or shake as necessary to initiate draining, and allow the separated mercury to rejoin that extending from the reservoir. The gas (bubble) will be compressed and pushed out to the wall of the throat at this point. It may or may not be visible, with or without magnification.

G. Turn the thermometer slightly towards the reversed position (about $10^{\circ}$ from horizontal) and again chill the reservoir in crushed ice. The slightly reversed angle will ensure that the bubble cannot move back toward the reservoir while not being sufficiently reversed to cause a new separation of the mercury at the bubble locus. The contracting mercury will move back into the reservoir past the compressed bubble, leaving it in situ.

H. When reasonably chilled (about 1 min.) fully reverse the instrument while coincidentally giving a knuckle tap to induce separation of the mercury at the bubble. (If the mercury does not wish to separate at the bubble but instead runs out of the appendix and separates at the break-off point, right the thermometer to rejoin the mercury and try again, this time with the UDSRT only partly reversed while giving a sharp, axial shake in the direction of the bulb; inertia should help the mercury to separate at the bubble.)

I. Now again heat the reservoir. After this second heating, the bubble will usually have been moved around the goose-neck bend so that it will be in the same position relative to the mercury extending from the reservoir as in a protected DSRT or a straight-shanked UDSRT and it can be moved on up into the bulb with the standard manipulation sequence described in section VI. If the bubble has not yet moved around the goose-neck bend, repeat operations $F, G$ and $H$.

XI. FAILURE TO DRAIN

In some DSRT's, the mercury will not drain from the bulb upon righting after reversal without some mechanical stimulus. This is inherent in the thermometer, possibly due to excessive evacuation of the capillary system or perhaps because of impurities in the mercury or in the glass of the bulb which cause chemical or molecular bonding or amalgamation which 
inhibits movement of the mercury out of the end of the bulb. Generally speaking, the lower the thermometer reading, the less "lead" of mercury into the stem from the bulb to pull it downward by gravity when the thermometer is righted and the more likely that mechanical stimuli will be needed to cause draining.

Such stimuli may be applied in several ways, depending upon the degree of reluctance of the mercury to leave the bulb:

A. Assuming that the upright DSRT is still in its tube on the bottle or reversing frame, first try lateral tapping of the tube with a solid object, such as a "messenger". If this is ineffective, tap the base of the tube from below; if there is only one DSRT on the bottle or frame, no damage to the appendix should result, since there will be little, if any, weight of mercury bearing on the appendix because most, or all, of the mercury above the appendix is suspended in the bulb and adjacent stem at the other end of the instrument. However, if there is more than one DSRT in the tubes on the bottle or frame, such tapping from below should not be done, since the other DSRT(s) in the tubes will presumably have drained properly and such axial tapping from below, with the full weight of the mercury column bearing on the appendix might seriously damage or ruin the drained instrument(s). In such a situation, the DSRT failing to drain should be removed from its tube for treatment individually.

B. With the non-draining DSRT now out of its tube, upright in the hands, shake it axially downward with some force and a sudden stop; this is the same motion as described in Section VI-E above.

C. If this maneuver is not effective in getting the mercury to move out of the bulb, the appendix must then be cleared of mercury before attempting the next, more violent maneuver so as to avoid possibility of damage to the appendix. With the DSRT horizontal, heat the reservoir until a substantial amount of mercury has expanded beyond the appendix into the pigtail (perhaps to near point "a" as described in section VI-A). Partly reverse the instrument and give it a sharp, axial shake downward or a tap from the bulb end, then shake the thermometer as necessary, utilizing the inertia of the free-floating mercury, to get the thus-separated portion of mercury partly around the pigtail so that it will not flow back down into the appendix when the thermometer is righted. Now right the instrument and cool the reservoir so that the mercury in the throat will be drawn back away from the appendix toward the reservoir.

D. With the appendix area now clear of mercury, DSRT upright, tap the reservoir end with some authority against a solid, wood surface. (This is the same maneuver, but in the opposite direction, as described in section VII-C above.) The amount of force permissible cannot be accurately described and one must use one's own best judgment, as stated earlier in Section VII-C; protected DSRT's will stand more force than UDSRT's. This action is generally effective, even with very uncooperative instruments. With such treatment, it is not uncommon for the mercury to separate in the throat of the bulb, and in any case, if a separation does occur, in or out of the bulb, it is important that any such separated mercury does not get all the way back into the pigtail until it can be determined where the separation occurred, so after each vertical strike of the DSRT, it should be turned at once to a horizontal position to halt temporarily further movement of the mercury in the capillary until such determination can be made. 
E. If, after such vertical striking, a separation of the mercury occurs a reasonable distance into the bulb, where the sectional area is substantially greater than in the bulb throat, it is now desirable to increase the mass of the body of mercury free in the stem by rejoining it with the mercury in the other end of the instrument. Partially right the thermometer and snap it, as one might a clinical thermometer (see Section VI-D) to drive the mercury free in the stem back into the pigtail. If it will not then meet and join with the body of mercury in the pigtail, it will be necessary to heat the reservoir, DSRT horizontal, until the expanding mercury from the reservoir rejoins that in the pigtail; continue heating until the mercury extending from the reservoir enters the beginning of the stem. Now reverse the thermometer, shake it axially if necessary to assist the mercury to leave the appendix and run down into the bulb, and then proceed to move the bubble in the bulb out to the end of the bulb as described in Section VI-E.

\section{MERCURY SEPARATED IN THROAT OF BULB} Occasionally, most commonly in DSRT's which exhibit difficulty in draining, upon righting after reversal (frequently subsequent to a deliberate tap) separation of the mercury will occur in the throat of the bu1b. This is a location which is often hidden by the metal, auxiliary mounting fitting, so it is not always possible to see exactly where the separation point is. This condition is very objectionable and often hard to reduce, as there is no "lead" of the mercury into the stem and the body of the bulb is totally full of mercury, with no void in which to work or knock off globules (Section VI-E). This condition can be the result of maneuvers described in the preceding section (XI). There are several techniques that can be used, or tried, to dislodge this stuck mercury from the bulb:

A. As recommended by Richter \& Wiese, this mercury in the bulb may be heated so as to cause it to expand into the stem, giving it a slight "lead" so that it will then respond to one of the treatments described in the previous section (XI). In a protected DSRT, this is a relatively slow process, as the shell and the air inside must also be heated in order to heat the mercury in the bulb. In addition, such heating must be limited to a temperature level which will not cause the auxiliary mercury to expand into the auxiliary expansion chamber where subsequent maneuvers might cause some of it to be knocked off and separated in the chamber.

B. In the case of a UDSRT, with the thermometer out of its she11, the mercury stuck in the bulb may be driven out by application of flame heat directly to the end of the bulb until some of the mercury near the tip vaporizes, driving the rest of the mercury in the bulb back into the stem toward and probably into the pigtail. This heating should be done with a small, moderate flame, using great care.

Hold the UDSRT nearly horizontal, slightly inclined toward the upright position $\left(5-10^{\circ}\right)$. Move the tip of the bulb quickly in and out of the edge of the flame, first on one side, then the other, alternately, rotating the UDSRT in the fingers back and forth something more than $180^{\circ}$ about its axis, so that the auxiliary reservoir is always away from and slightly below the flame with each pass of the main bulb through the flame edge. The trick is to be sure not to heat the bulb tip so rapidly that it 
might crack. As it gets hotter and hotter with each pass through the flame edge, on alternate sides, the speed of the passes through the flame may be slowed, so that with each pass the bulb is in the flame envelope a little 1onger. (We are talking about intervals of a few hundredths of a second initially, up through tenths to eventually a second or more, with intervals out of the flame routinely of several seconds duration.) Eventually the glass of the bulb tip will get hot enough to vaporize some of the mercury inside $\left(358^{\circ} \mathrm{C}\right)$ which will "blow" the rest of the mercury out of the bulb.

As soon as the vaporization occurs, stop the heating and very carefully set the UDSRT down, horizontal, and allow it to cool slowly, without inducement, until the auxiliary mercury, which may possibly have expanded into the expansion chamber during the process of heating the main bulb, has contracted entirely into the auxiliary stem capillary. If the thermometer is jarred while auxiliary mercury is in the expansion chamber, some of it may get knocked off and separated, which would cause a secondary problem.

Now right the thermometer and be sure that all the main mercury is continuous from the reservoir. If it is not, heat the reservoir as needed to rejoin the mercury from the reservoir with that loose in the pigtail area. Reverse the thermometer to check for proper functioning. If the mercury breaks correctly, all is well. If the mercury separated somewhere else, presumably in the pigtail, this is probably caused by a bubble which may have been the cause of the separation in the throat of the bulb in the first place. Proceed to remove the bubble as described in Section VI.

C. Obviously, the direct application of flame heat described in the preceding paragraphs cannot be used with a protected DSRT. However, it is possible to generate enough heat in the end of a protected bulb, inside the she11, to vaporize mercury by use of a 3-4X magnifying glass (1ens) 3 $1 / 2-4^{\prime \prime}$ in diameter, using the sun as a heat source.

To set up for this operation, one needs a chemical apparatus stand or equivalent and assorted laboratory clamps to hold the magnifying lens and DSRT in such manner that the lens is at right angles to the sun's rays with the focal point of the concentrated rays on the end of the bulb cavity and a means of holding the stand, with its attachments, in alignment with the sun. It is helpful in making the focal and alignment adjustments to cover the lens with a relatively dense acetate or glass filter, preferably red, during such setting-up process so that the focal point can be observed with the naked eye without injury. Once the set-up is made, one must use double density (or equivalent) brazing-cutting goggles so as to be able to observe the end of the bulb (focal point) without damage to the eyes which would certainly result otherwise due to the extreme intensity of unfiltered light at the focal point.

With the bright sun fairly low in the sky (shortly before noon in mid-February at Lat. $41^{\circ} \mathrm{N}$ ) this operation has been tested and it works. Under such conditions, because the light in the cone of concentration is refracted and partly reflected by the glass of the shell and also by the glass of the bulb, the rate of heating of the bulb will not be so great as to crack the glass of the bulb. If the sun were more nearly overhead, possibly some initial filtering or intermittent interuption of the sunlight entering the magnifying lens might be needed. When properly set up (sun 
focused on the mercury in the end of the bulb) only a few minutes should be needed to raise the temperature of this mercury to the vaporizing point. (In the tests cited above, about five minutes were needed, and the auxiliary temperature had risen to only $51^{\circ} \mathrm{C}$ ).

Conceivably one could generate enough heat to accomplish this vaporization by use of a high-energy artificial light source, such as an electrical arc lamp. No experiments have been made along these lines.

\section{EXCESSIVE GAS IN DSRT SYSTEM}

On rare occasions, there may be encountered a DSRT which is reliable at temperatures where readings occur one-third or more up the length of the stem but which begin to become erratic as readings occur lower down in the stem nearer the bulb. The cause is almost certainly excessive gas (insufficient evacuation) in the capillary system. With such condition, at temperatures giving readings low in the stem there is not enough weight of mercury standing up in the stem to compress to nullity the gas in the end of the bulb, so that the mercury extending up from the bulb is riding on a cushion of gas and will not set down solidly in the bulb. In this case, it is often impossible to obtain a valid ice point, as successive readings at $0 .{ }^{\circ} \mathrm{C}$ will vary up to half a graduation interval or more.

Valid and consistent ice point readings can sometimes be obtained by tapping the DSRT axially from the bottom after reversal to seat the mercury in the bulb. In such case, the DSRT is usable but its use should be confined to situations in which readings will appear in the upper two-thirds of the stem, where there will be enough weight of mercury above the gas in the end of the bulb to compress it totally.

When excessive gas is suspected of being present and the cause of erratic readings, a simple test will confirm its presence. Reverse the DSRT at a temperature where the reading will be about mid-way in the stem. While observing this reading (top of the mercury column) rotate the instrument slowly back toward horizontal; if excessive gas is present, as the rotation progresses the top of the mercury column will be seen to move upward, back toward the reservoir; in an attitude near horizontal, this movement may have progressed a substantial distance along the stem and there will probably be a visible void (bubble of expanding gas) in the end of the bulb.

There is no cure for this condition other than the possible rebuilding and evacuation of the instrument by the manufacturer (if he will undertake it) but such instruments can be used for temperatures showing in the upper two-thirds of the stem, provided satisfactory ice point checks can be made periodically.

XIV. SEPARATED AUXILIARY MERCURY

The auxiliary thermometer of a DSRT is not evacuated as is the main thermometer but has in its capillary a certain amount of gas. The purpose of this is to keep the auxiliary mercury continuous and immobile, except for expansion and contraction with temperature change, so that it cannot run freely in the capillary when the DSRT is upright.

Occasionally, usually as the result of transporting or handling a DSRT upright in a temperature low enough to contract the auxiliary mercury entirely into the auxiliary reservoir, some of the gas which is in the 
auxiliary capillary will enter the auxiliary reservoir and it will almost certainly then move, as a bubble, into the far (now uppermost) end of the auxiliary reservoir. If the DSRT is then brought to a warmer temperature, the auxiliary mercury will expand back into the capillary, with the errant gas still in the end of the auxiliary reservoir. If the DSRT is then reversed, slight jarring or vibration will cause this gas to move up to the capillary end of the auxiliary reservoir and enter the capillary of the stem. With further warming, this gas will be driven entirely out of the auxiliary reservoir by the expanding mercury and a separation of the auxiliary mercury column will occur. The size of the separation will depend upon the volume of gas which had entered the auxiliary reservoir and also upon the actual temperature, since the gas itself will expand as it is heated and occupy more volume.

Because of this possibility, if a DSRT is to be transported or stored in the extremely low temperatures referred to in the previous paragraph, they should be kept in the reversed position, contrary to the normally desirable upright position for less extreme cold. When using DSRT's at sea in such extreme cold, they should be stored inside the cabin, not out on deck. In this manner, when making a cast, the bottles or reversing frames, with their DSRT's, are brought out unchilled, put immediately on the wire and then promptly lowered into the relative warmth of the water, before the auxiliaries have cooled excessively. After retrieval of the cast, the DSRT's should be taken promptly back into the cabin for reading and subsequent righting to prepare for the next cast. DSRT's should not be left reversed once the reading and withdrawal of water samples has been completed. (See Section $\mathrm{V}_{0}$ )

There are two approaches to rejoining a separated mercury column of an auxiliary thermometer. (These apply generally to any simple, mercury-in-glass thermometer.)

1. With the DSRT reversed, super-chill the auxiliary reservoir to near the freezing (solidifying) point of mercury $\left(-38^{\circ} \mathrm{C}\right.$ ) using the alcohol-dry ice technique described in Section IX. With a UDSRT, do not put the entire auxiliary reservoir in the bath, so that the mercury in the stem end of the auxiliary reservoir cannot freeze. (See note under Section IX-H). Lateral tapping of the lower end of the instrument during this chilling should ensure that all of the gas in the auxiliary reservoir will have moved up toward the capillary, above all the mercury in the auxiliary reservoir. (The separated portion of mercury in the stem will not voluntarily enter the reservoir, since it is effectively sandwiched or floating between two cushions of gas, one above, one below.) Now hold the DSRT firmly by the (main) reservoir end and snap it rapidly downward through an arc of $90^{\circ}$ or more; centrifugal force should drive the mercury remaining in the stem to move down into the auxiliary reservoir. If the capillary is now entirely clear of mercury, the gas in the auxiliary reservoir is now presumably above all the mercury.

Allow the auxiliary to warm (DSRT still reversed) until the auxiliary mercury enters the capillary, then check for absence of gas or separation by heating the auxiliary reservoir until the mercury has expanded the full length of the auxiliary stem, but not into the expansion chamber. The mercury column of the auxiliary should be continuous. 
2. If all the mercury in the auxiliary stem cannot be made to enter the auxiliary reservoir as just described, elimination of the bubble and rejoining of mercury must be accomplished at the other end of the auxiliary in the expansion chamber. (Note that the procedure described in paragraph 1 above should be carried out first in any case, so as to leave as little mercury as possible above the gas in the reservoir.)

With the DSRT still reversed, heat the auxiliary reservoir so as to drive the bubble and mercury above it up into the expansion chamber. This may require a temperature of $100^{\circ} \mathrm{C}$ or more. When the end of the principal body of mercury extending from the auxiliary reservoir has reached the expansion chamber, the gas which had caused the separation will be pushed out to the side into the expansion chamber where it belongs. If this does not happen voluntarily, tip the reversed DSRT $30^{\circ}$ or so toward horizontal and tap the instrument in the area of the expansion chamber from below with a knuckle to dislodge the bubble and rejoin the separated portions of mercury. Keep the DSRT reversed and allow the auxiliary to cool until all the auxiliary mercury has contracted out of the expansion chamber and back into the stem. If the bubble tries to re-enter the stem during this cooling, still leaving a separation, reheat the auxiliary and try again; eventually the procedure will be successful.

A separation of the auxiliary mercury may occasionally occur in the expansion chamber as the result of a jar of the DSRT while the auxiliary is sensing a temperature high enough to have expanded the auxiliary mercury partly into the expansion chamber. Such a jar may knock loose one or more globules of mercury into the chamber.

When this has occurred, with the DSRT reversed heat the auxiliary reservoir enough to expand the mercury up into the beginning of the expansion chamber. Tap the DSRT laterally in the area of the expansion chamber or axially from the bottom, as appropriate, to move the separated mercury globule(s) down the wall of the expansion chamber to rejoin with the mercury extending up the stem. Cool the auxiliary as just described above.

If a bit of mercury, possibly so small as to be barely visible, has been separated in the auxiliary expansion chamber and somehow migrated to the far end of the chamber, it may be very difficult to dislodge by tapping. If a few, relatively firm lateral taps do not move the bit of mercury out of the tip of the expansion chamber, it is best to forget it and leave it there, as it is unlikely to be dislodged by normal use of the DSRT, and excessive violence in attempts to move such resident mercury may result in breakage. In such case, an ice point check must be made to determine the new auxiliary ice point reading, which will be lower than previously by the volume of the bit of mercury presumably permanently lost in the expansion chamber.

Occasionally with age, molecules of the auxiliary mercury may start to adhere to the capillary wall, presumably due to impurities. (This can happen with any mercury-in-glass thermometer.) The results are darkened areas of the capillary, making readings in such areas impossible and an ice point reading lowered by the volume of mercury adhered to the capillary wall above the $0 .{ }^{\circ} \mathrm{C}$ point. Readings above the area of mercury adhesion will be unaffected, as the adhered mercury has again become part of the total volume of mercury in the system. There is no cure for this condition and when it becomes bad enough to affect the accuracy of data obtained with the DSRT, the auxiliary should be replaced. 
Whenever it is necessary to replace an auxiliary for any reason, it is economical of time and effort if it can be replaced with an auxiliary canibalized from a broken or otherwise defective DSRT, the calibration data of which is known. In this way, after replacement it is only necessary to do an ice point check instead of a complete auxiliary calibration.

XV. SUMMARY

In summary, the following points concerning the care, treatment, storage, use, function, malfunction, damage, manipulation, etc. should be kept in mind. (For calibration of DSRT's, see W.H.O.I. Technical Report 非6-7, "Equipment and Procedures for the Calibration of Deep Sea Reversing Thermometers" by G. G. Whitney, Jr., February 1976.)

1. DSRT's are delicate instruments and can be easily broken. Handle with care.

2. When DSRT's are in a water bottle or reversing frame, never trip the bottle or frame reversing mechanism in air; breakage of the DSRT's can easily result. The reversing mechanisms of such devices carrying DSRT's must be tripped only in water.

3. DSRT's should always be transported and stored in the upright position EXCEPT in extreme cold. (See Section XIV.)

4. If an apparent malfunction has occurred, manipulate first for a gas bubble (Section VI). Sometimes several manipulation sequences may be needed to eliminate all gas from within the mercury column. Be patient.

5. Never strike an upright DSRT axially from the bottom while any significant amount of mercury is in and above the appendix. Doing so may ruin the instrument. (Section VII).

6. Some authorities recommend "exercising" (reversing) DSRT's daily. While this is certainly beneficial, in the author's opinion it is not necessary and if the organization's roster of DSRT's consists of many hundred, such exercise becomes a rather major undertaking. To get around this objection, elaborate and expensive racks have been built to store and exercise groups of perhaps up to 50 DSRT's, with perhaps half a dozen such groups to a machine. While this eliminates the need to handle each DSRT individually, each one must still be inspected with a reading glass to see that normal function has occurred, with mercury leaving the appendix empty and having broken off at the break-off point. If any of the instruments have malfunctioned, they must be removed and manipulated to restore proper function. Failure to do this effectively negates the object of the "exercise".

In the author's opinion, a few reversals a year are adequate exercise and most DSRT's will get more than this through periodic use at sea. After having stood upright, unexercised for a long period, if the mercury does not flow freely out of the appendix and break properly when the instrument is first reversed, a tap or two, laterally or axially as described in Section VII-A and $\mathrm{C}$, should loosen the mercury and restore normal functioning. In such cases, a number of successive reversals should then be made in hand, with heat of one hand warming the reservoir slightly, to verify that normal functioning is now occurring regularly. 
XVI. REFERENCES AND ACKNOWLEDGEMENTS

G. G. Whitney, Jr., "Notes on Malfunctional Behavior and its Correction in Deep Sea Reversing Thermometers", Woods Hole Oceanographic Institution, Technical Report No. 52-29, May, 1952.

To Karin Bohr, for her excellent typing and proof reading of the necessarily very precise and tedious instructions and to W. G. Metcalf, who so carefully read the original draft manuscript and made many helpful editorial comments.

To F. Medeiros, Cynthia Moor and other members of D. Souza's Graphic Arts staff who so expertly made photographic enlargements and reductions after various delicate alterations and embellishments of Figure 1 and ultimately organized, printed and bound the whole to make this volume.

To Richter and Wiese, Berlin and Watanabe Keiki Mfg. Co., Tokyo, whose line drawings were used as the basis for those in Figure 1. 


\begin{tabular}{|c|c|c|}
\hline $\begin{array}{l}\text { BIBLIOGRAPHIC DATA } \\
\text { SHEET }\end{array}$ & $\begin{array}{l}\text { 1. Report No. } \\
\text { WHOI }-79-68\end{array}$ & 3. Recipient's Accession No. \\
\hline \multirow{2}{*}{\multicolumn{2}{|c|}{$\begin{array}{l}\text { 4. Title and Subtitle } \\
\text { DIAGNOSIS AND CORRECTION OF MALFUNCTIONAL BEHAVIOR } \\
\text { IN DEEP SEA REVERSING THERMOMETERS }\end{array}$}} & $\begin{array}{l}\text { 5. Report Date } \\
\text { August } 1979\end{array}$ \\
\hline & & 6. \\
\hline $\begin{array}{l}\text { 7. Author(s) } \\
\text { G. G. Whitnes }\end{array}$ & Jr. & $\begin{array}{l}\text { 8. Performing Organization Rept. } \\
\text { No. WHOI }-79-68\end{array}$ \\
\hline \multirow{2}{*}{\multicolumn{2}{|c|}{$\begin{array}{l}\text { 9. Performing Organization Name and Address } \\
\text { Woods Hole Oceanographic Institution } \\
\text { Woods Hole, MA } 02543\end{array}$}} & 10. Project/Task/Work Unit No. \\
\hline & & 11. Contract/Grant No. \\
\hline \multirow{2}{*}{\multicolumn{2}{|c|}{$\begin{array}{l}\text { 12. Sponsoring Organization Name and Address } \\
\text { Woods Hole Oceanographic Institution } \\
\text { Woods Hole, MA } 02543\end{array}$}} & \begin{tabular}{|l}
$\begin{array}{l}\text { 13. Type of Report \& Period } \\
\text { Covered } \\
\text { Technical } \quad(1951- \\
1979) \\
\end{array}$
\end{tabular} \\
\hline & & 14. \\
\hline \multicolumn{3}{|l|}{ 15. Supplementary Notes } \\
\hline \multicolumn{3}{|c|}{$\begin{array}{l}\text { The nomenclature, morphology and functioning of deep sea reversing } \\
\text { thermometers are identified and explained. The various types of mal- } \\
\text { functions, nonfunctions and other abberations are defined and detailed } \\
\text { instructions are given for the procedures used to restore normal } \\
\text { functioning, to the extent possible. Advice is given regarding the use, } \\
\text { handling, storage and transportation of these instruments so as to } \\
\text { avoid damage and actions which might cause malfunctions to occur. }\end{array}$} \\
\hline \multicolumn{3}{|c|}{ 17. Key Words and Document Analysis. 17a. Descriptors } \\
\hline
\end{tabular}

17b. Identifiers/Open-Ended Terms

17 c. COSATI Field/Group

\begin{tabular}{|c|c|c|}
\hline \multirow[t]{2}{*}{ 18. Availability Statement } & $\begin{array}{l}\text { 19. Security Class (This } \\
\text { Report) } \\
\text { UNCLASSIFIED }\end{array}$ & $\begin{array}{c}\text { 21. No. of Pages } \\
27\end{array}$ \\
\hline & $\begin{array}{l}\text { 20. Security Class (This } \\
\text { Page } \\
\text { UNCLASSIFIED }\end{array}$ & 22. Price \\
\hline
\end{tabular}




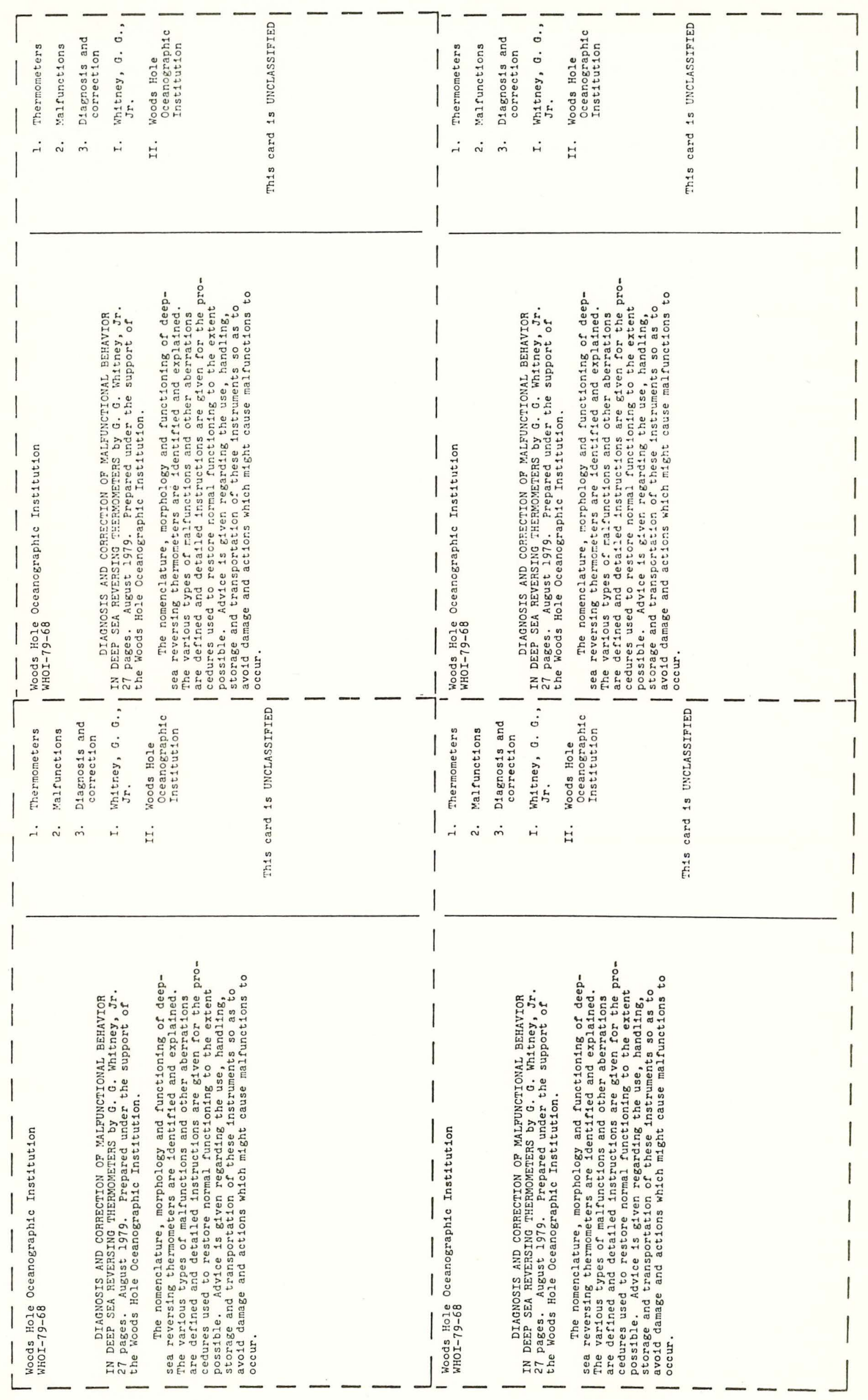

